\title{
Linear and Weakly Nonlinear Energetics of Global Nonhydrostatic Normal Modes
}

\author{
Carlos F. M. Raupp, André S. W. Teruya, and Pedro L. Silva Dias \\ Instituto de Astronomia, Geofísica e Ciências Atmosféricas, Universidade de São Paulo, São Paulo, Brazil
}

(Manuscript received 14 May 2019, in final form 28 August 2019)

\begin{abstract}
Here the theory of global nonhydrostatic normal modes has been further developed with the analysis of both linear and weakly nonlinear energetics of inertia-acoustic (IA) and inertia-gravity (IG) modes. These energetics are analyzed in the context of a shallow global nonhydrostatic model governing finite-amplitude perturbations around a resting, hydrostatic, and isothermal background state. For the linear case, the energy as a function of the zonal wavenumber of the IA and IG modes is analyzed, and the nonhydrostatic effect of vertical acceleration on the IG waves is highlighted. For the nonlinear energetics analysis, the reduced equations of a single resonant wave triad interaction are obtained by using a pseudoenergy orthogonality relation. Integration of the triad equations for a resonance involving a short harmonic of an IG wave, a planetary-scale IA mode, and a short IA wave mode shows that an IG mode can allow two IA modes to exchange energy in specific resonant triads. These wave interactions can yield significant modulations in the dynamical fields associated with the physical-space solution with periods varying from a daily time scale to almost a month long.
\end{abstract}

\section{Introduction}

Atmospheric normal mode function theory has long received considerable research effort due to not only the central role of normal modes in theoretical toy models designed to elucidate mechanisms of the atmospheric dynamics that are not clearly distinguishable in full atmospheric general circulation models (AGCMs), but also the practical applications of normal modes in data assimilation (Žagar et al. 2004) and initialization of numerical weather prediction models (Baer and Tribbia 1977; Machenhauer 1977; Leith 1980). A review of research on normal modes of atmospheres over spheres can be found in Kasahara (2004). In addition, the normal mode functions of threedimensional primitive equations in spherical coordinates can also be utilized in diagnostic analysis of AGCM outputs by projecting global circulation dynamical fields onto these eigenfunctions (see, for instance, Kasahara and Puri 1981; Žagar et al. 2015).

The normal modes of a dynamical system represent its free small amplitude oscillations around a reference steady state and are obtained as eigensolutions of the linearized version of the corresponding partial differential equations. For the atmosphere, this reference

Corresponding author: Dr. Carlos F. M. Raupp, carlos.raupp@ iag.usp.br steady state is usually defined as a motionless and stably stratified atmosphere, since in this case the leadingorder wave actions by which the orthogonality relations hold are positive definite integrals, represented by either pseudoenergy or pseudomomentum (Shepherd 1990; Vallis 2006), and consequently the eigensolutions represent purely stable wave motions. In this context, the effect of nonlinearity is to break down the independence of the normal modes, allowing their energy to be exchanged within triads of resonant eigenmodes (see Raupp et al. 2008, and references therein).

Due to the extensive use of global numerical models with coarse resolutions until the 1990s, the research effort on atmospheric normal mode functions has mostly focused on global hydrostatic primitive-equation models (Longuet-Higgins 1968; Kasahara 1976, 1977, 1978), as well as their equatorial beta-plane (Matsuno 1966; Lindzen 1967; Silva Dias et al. 1983; DeMaria 1985; Raupp et al. 2008; Raupp and Silva Dias 2009) and quasigeostrophic (Vallis 2006; Pedlosky 1987, and references therein) approximations. Nevertheless, with the increasing utilization of high-resolution numerical models during recent decades, there has been a renewed interest on normal mode function theory of global nonhydrostatic models. Kasahara and Qian (2000, hereafter referred to as KQ00) studied the linear theory of nonhydrostatic normal modes of a shallow global nonhydrostatic model 
in spherical coordinates, while Qian and Kasahara (2003) performed a similar analysis for the equatorial and midlatitude beta planes. The shallow nonhydrostatic model adopted in these studies considers the traditional approximation (Phillips 1973) and neglects all the apparent acceleration terms involving the vertical velocity in the horizontal momentum equations, as well as all the apparent acceleration terms of the vertical momentum equation.

In this context, Kasahara $(2003 \mathrm{~b}, \mathrm{a})$ augmented the linear theory of nonhydrostatic normal modes developed in KQ00 and Qian and Kasahara (2003) by accounting for the nontraditional Coriolis terms proportional to the cosine of latitude in the momentum equations in the context of the tangent plane geometry. He analyzed the role of these nontraditional terms in the dispersion relation of inertia-gravity (IG) and inertia-acoustic (IA) modes. Kasahara (2004) computed the normal modes of the full deep nonhydrostatic global model, including all the curvature and nontraditional Coriolis terms of the momentum conservation in spherical coordinates, along with not performing the traditional approximation. Unlike the hydrostatic primitive equations and the shallow nonhydrostatic model, in the deep nonhydrostatic model in spherical coordinates the linearized equations around a resting basic state are no longer separable into two simultaneous eigenvalue problems representing the vertical and horizontal structures of the normal modes. Therefore, Kasahara (2004) proposed a numerical procedure involving a finitedifference discretization in the vertical direction and the solution of the meridional eigenvalue problem for each vertical level. He then compared the solutions of the deep nonhydrostatic model with those of the shallow nonhydrostatic model obtained by KQ00.

Apart from analyzing the linear theory of nonhydrostatic normal modes, all the studies cited above have concerned only on their dispersion relation (eigenfrequencies). Therefore, here we aim to further extend the understanding on the global atmospheric nonhydrostatic normal modes by analyzing both the linear and weakly nonlinear energetics of the eigenmodes of the shallow nonhydrostatic model. In the former case, we have computed the mode energies derived by KQ00 and analyzed the energetics of inertia-gravity and inertia-acoustic modes as a function of the zonal wavenumber. As shall be demonstrated later, this analysis clearly elucidates the important role of the nonhydrostatic effect on the internal gravity waves for very large zonal wavenumbers. In the latter case, we have investigated the possibility of resonant interactions involving very short inertia-gravity wave modes (whose zonal wavenumber $s>400$ ), which are strongly affected by the nonhydrostatic effect of vertical acceleration in the present model, and inertia-acoustic modes, which are exclusively characteristic of nonhydrostatic dynamics.
Therefore, the present paper can be regarded as an extension of previous works on global nonhydrostatic normal modes, especially with our analysis of the nonlinear dynamics of these modes. Also, our study may be considered as an extension of previous investigations on the nonlinear dynamics of atmospheric normal modes (i.e., Vanneste and Vial 1994; Raupp et al. 2008; Ripa 1983a,b; Domaracki and Loesch 1977; Loesch and Deininger 1979; Lynch 2009) by accounting for the nonhydrostatic effects, since these studies have investigated the nonlinear atmospheric normal modes in the hydrostatic context. Their results shall be appropriately cited throughout sections 3 and 5. The results of our weakly nonlinear analysis on nonhydrostatic normal modes may also open a new possible research line to further understand the hydrostatic adjustment problem in the atmosphere, as will be discussed in section 6 .

The remainder of this paper is organized as follows. In section 2, we present the model equations and the perturbation theory adopted. Section 2 also revisits the linear theory of global nonhydrostatic eigenmodes developed by KQ00 for the shallow global nonhydrostatic model. In section 3 we have described the general theoretical framework regarding pseudoenergy conservation and its spectral representation in terms of normal modes. In section 4 we have computed the intrinsic energy of the eigenmodes (i.e., their Hilbert norm) and analyzed its dependence upon the zonal wavenumber for IA and IG wave types. Section 5 explores the weakly nonlinear energetics of the normal modes by analyzing the possibility of resonant interactions involving IA and IG modes. Section 6 summarizes the main conclusions and discusses the implications of the results for the hydrostatic adjustment problem in the atmosphere, as well as the sensitivity of the results of our weakly nonlinear analysis to the upper boundary condition adopted here.

\section{Model equations and perturbation method}

\section{a. Shallow global nonhydrostatic model}

The model adopted here is the so-called shallow global nonhydrostatic model (White et al. 2005). This model can be obtained from the full Euler equations in a uniformly rotating frame and spherical-oblate coordinate system by approximating the geopotential surfaces by spheres ${ }^{1}$ and adopting some approximations that result from classical scale analysis for large-scale

\footnotetext{
${ }^{1}$ This approximation alone yields the so-called deep nonhydrostatic model (White et al. 2005).
} 
motions in the atmosphere. These approximations include (i) neglecting the apparent acceleration terms proportional to the vertical velocity in the horizontal momentum equations, including both those associated with the curvature of the coordinate system and the Coriolis term proportional to the cosine of latitude in the zonal momentum equation, and (ii) disregarding all the apparent acceleration terms in the vertical momentum equation. Furthermore, the approximation of a shallow atmosphere assumes that its thickness is very small compared to Earth's radius, which is regarded as a constant. Under these considerations, we can express the equations of momentum, mass continuity, thermodynamics for adiabatic motions of dry air, as well as the equation of state, as follows:

$$
\begin{aligned}
\frac{D u}{D t}-\left(f+\frac{u}{a} \tan \phi\right) v+\frac{1}{\rho a \cos \phi} \frac{\partial p}{\partial \lambda} & =0, \\
\frac{D v}{D t}+\left(f+\frac{u}{a} \tan \phi\right) u+\frac{1}{a \rho} \frac{\partial p}{\partial \phi} & =0, \\
\frac{D w}{D t}+\frac{1}{\rho} \frac{\partial p}{\partial z}+g & =0, \\
\frac{D \rho}{D t}+\rho\left(\nabla \cdot \mathbf{V}+\frac{\partial w}{\partial z}\right) & =0, \\
\frac{D p}{D t}-\gamma R T \frac{D \rho}{D t} & =0, \\
p-\rho R T & =0 .
\end{aligned}
$$

In the equations above, all the variables, symbols and operators have their usual meanings and are displayed in Table 1. Recall that classical scale analysis of the deep nonhydrostatic model for synoptic-scale motions leads to the hydrostatic primitive-equation model, since the vertical acceleration term in (3) is about $10^{6}$ times smaller in magnitude than the remaining terms of this equation for these kinds of motions (e.g., Holton 2004). Nevertheless, KQ00 utilized the same model equations, (1)-(6), as the simplest context to study the normal modes of global nonhydrostatic models. In fact, (1)-(6) differ from the hydrostatic primitive-equation model only by this vertical acceleration term in (3), and therefore consist of a small relaxation of the hydrostatic primitive-equation model (White et al. 2005). As a result, for example, the effect of this nonhydrostatic term on the inertia-gravity waves only becomes evident for very short harmonics having zonal wavenumber higher than 400 (KQ00), as will be shown later. Therefore, as we aim here to extend KQ00's study on global nonhydrostatic normal modes by investigating the modes' energetics, we shall adopt the same perturbation theory considered in KQ00 for (1)-(6), but focusing on their particular case of an isothermal background atmosphere. Nevertheless, due to our nonlinear analysis in section 5, unlike KQ00 we will include the quadratic terms in perturbation amplitude, which correspond to the leading-order nonlinear terms in the context of small amplitude perturbations.

\section{b. Perturbation theory}

To analyze the normal modes of system (1)-(6) we consider small amplitude perturbations superimposed on a resting, hydrostatic and isothermal background state; that is,

$$
\begin{aligned}
& u(\lambda, \phi, z, t)=u_{0}+u^{\prime}(\lambda, \phi, z, t), \\
& v(\lambda, \phi, z, t)=v_{0}+v^{\prime}(\lambda, \phi, z, t), \\
& w(\lambda, \phi, z, t)=w_{0}+w^{\prime}(\lambda, \phi, z, t), \\
& p(\lambda, \phi, z, t)=p_{0}(z)+p^{\prime}(\lambda, \phi, z, t), \\
& \rho(\lambda, \phi, z, t)=\rho_{0}(z)+\rho^{\prime}(\lambda, \phi, z, t), \\
& T(\lambda, \phi, z, t)=T_{0}+T^{\prime}(\lambda, \phi, z, t),
\end{aligned}
$$

with

$$
u_{0}=v_{0}=w_{0}=0, \quad T_{0}=\text { const }, \quad \text { and } \quad \frac{d p_{0}}{d z}=-\rho_{0} g .
$$

The isothermal assumption for the undisturbed state implies that

$$
\begin{aligned}
& \rho_{0}(z)=\rho_{0}(0) e^{-z / H}, \\
& p_{0}(z)=p_{0}(0) e^{-z / H},
\end{aligned}
$$

where $H=R T_{0} / g$ corresponds to the scale height of the isothermal atmosphere. Substituting (7)-(13) into (1)(6) and retaining the terms up to second order in terms of perturbations yield

$$
\begin{aligned}
\frac{\partial u^{\prime}}{\partial t}-f v^{\prime}+\frac{1}{\rho_{0} a \cos \phi} \frac{\partial p^{\prime}}{\partial \lambda}= & -\mathbf{V}^{\prime} \cdot \nabla u^{\prime}-w^{\prime} \frac{\partial u^{\prime}}{\partial z} \\
& +\frac{u^{\prime} v^{\prime}}{a} \tan \phi+\frac{\rho^{\prime}}{\rho_{0}^{2} a \cos \phi} \frac{\partial p^{\prime}}{\partial \lambda}, \\
\frac{\partial v^{\prime}}{\partial t}+f u^{\prime}+\frac{1}{\rho_{0} a} \frac{\partial p^{\prime}}{\partial \phi}= & -\mathbf{V}^{\prime} \cdot \nabla v^{\prime}-w^{\prime} \frac{\partial v^{\prime}}{\partial z} \\
& -\frac{u^{\prime 2}}{a} \tan \phi+\frac{\rho^{\prime}}{\rho_{0}^{2} a} \frac{\partial p^{\prime}}{\partial \phi}
\end{aligned}
$$


TABLE 1. Definition of all variables, symbols, and operators.

\begin{tabular}{ll}
\hline \hline \multicolumn{1}{c}{ Symbol } & \multicolumn{1}{c}{ Description } \\
\hline$\lambda$ & Longitude \\
$\phi$ & Latitude \\
$z$ & Height above Earth's surface \\
$t$ & Time \\
$u$ & Zonal wind field \\
$v$ & Meridional wind field \\
$w$ & Vertical wind field \\
$p$ & Pressure field \\
$\rho$ & Density field \\
$T$ & Temperature field \\
$a$ & Earth's radius $(=6370 \mathrm{~km})$ \\
$g$ & Gravity acceleration, assumed constant $\left(=9.8 \mathrm{~m} \mathrm{~s}^{-2}\right)$ \\
$f=2 \Omega \sin \phi$ & Coriolis parameter \\
$\Omega$ & Earth's rotation rate $\left(=2 \pi / 86400 \mathrm{~s}^{-1}\right)$ \\
$R$ & Gas constant for dry air $\left(=287 \mathrm{~J} \mathrm{~K} \mathrm{Kg}^{-1}\right)$ \\
$C_{p}$ & Thermal capacity at constant pressure $\left(=1004 \mathrm{~J} \mathrm{~K} \mathrm{~K}^{-1} \mathrm{~kg}^{-1}\right)$ \\
$C_{v}$ & Thermal capacity at constant volume $\left(C_{v}=C_{p}-R\right)$ \\
$\gamma$ & = $C_{p} / C_{v}$ \\
$\kappa$ & - R/C $C_{p}$ \\
$\mathbf{V}=(u, v)^{\mathrm{T}}$ & Horizontal velocity vector field \\
$D / D t=\partial / \partial t+\mathbf{V} \cdot \nabla+w \partial / \partial z$ & Material derivative, where $\mathbf{V} \cdot \nabla=[u /(a \cos \phi)](\partial / \partial \lambda)+(v / a)(\partial / \partial \phi)$ \\
$\nabla \cdot \mathbf{V}=[1 /(a \cos \phi)][\partial u / \partial \lambda+\partial(v \cos \phi) / \partial \phi]$ & Divergence of horizontal velocity \\
\hline
\end{tabular}

$$
\begin{aligned}
& \frac{\partial w^{\prime}}{\partial t}+\frac{1}{\rho_{0}}\left(\frac{\partial p^{\prime}}{\partial z}+\frac{g}{C_{s}^{2}} p^{\prime}-\theta^{\prime}\right)=-\mathbf{V}^{\prime} \cdot \nabla w^{\prime}-w^{\prime} \frac{\partial w^{\prime}}{\partial z} \\
& +\frac{\rho^{\prime}}{\rho_{0}^{2}} \frac{\partial \rho^{\prime}}{\partial z}+g\left(\frac{\rho^{\prime}}{\rho_{0}}\right)^{2}, \\
& \frac{1}{C_{s}^{2}} \frac{\partial p^{\prime}}{\partial t}-\frac{\rho_{0} g}{C_{s}^{2}} w^{\prime}+\rho_{0}\left(\nabla \cdot \mathbf{V}^{\prime}+\frac{\partial w^{\prime}}{\partial z}\right) \\
& =-\frac{1}{C_{s}^{2}}\left(\mathbf{V}^{\prime} \cdot \nabla p^{\prime}+w^{\prime} \frac{\partial p^{\prime}}{\partial z}\right)-\rho^{\prime}\left(\nabla \cdot \mathbf{V}^{\prime}+\frac{\partial w^{\prime}}{\partial z}\right) \\
& +\frac{1}{C_{s}^{2}} \frac{T^{\prime}}{T_{0}}\left(\frac{\partial p^{\prime}}{\partial t}-\rho_{0} g w^{\prime}\right), \\
& \frac{\partial \theta^{\prime}}{\partial t}+\rho_{0} N^{2} w^{\prime}=-\left(\mathbf{V}^{\prime} \cdot \nabla \theta^{\prime}+w^{\prime} \frac{\partial \theta^{\prime}}{\partial z}\right) \\
& +\frac{g}{C_{s}^{2}} \frac{T^{\prime}}{T_{0}}\left(\frac{\partial p^{\prime}}{\partial t}-\rho_{0} g w^{\prime}\right) .
\end{aligned}
$$

In the equations above, $C_{s}=\sqrt{\gamma R T_{0}}$ refers to the linear sound wave speed and the variable $\theta^{\prime}$ is related to entropy perturbation, given by

$$
\theta^{\prime}=\frac{g}{C_{s}^{2}} p^{\prime}-g \rho^{\prime}
$$

Furthermore, the perturbation temperature in (19) and (20) can be obtained from the linearized version of the equation of state:

$$
\frac{p^{\prime}}{p_{0}}=\frac{\rho^{\prime}}{\rho_{0}}+\frac{T^{\prime}}{T_{0}}
$$

The parameter $N$ appearing in (20) refers to the BruntVäisälä frequency, defined according to

$$
N^{2}=-g\left(\frac{d \ln \rho_{0}}{d z}+\frac{g}{C_{s}^{2}}\right)=\frac{\kappa g}{H},
$$

where the second equality holds for the special case of an isothermal atmosphere considered here.

\section{c. Linear eigenmodes}

KQ00 solved the linearized version of (16)-(20) subjected to a rigid lid vertical boundary condition, ${ }^{2}$

$$
w^{\prime}=0 \quad \text { at } \quad z=0 \text { and } z=z_{T}
$$

apart from requiring (i) zonal periodicity and (ii) regularity of the solutions at the poles. The eigensolutions of

\footnotetext{
${ }^{2}$ The upper boundary condition in (24) is a simplified model for tropospheric wave disturbances in which the coupling with the stratosphere is ignored. Nevertheless, this assumption is important in our simplified context of an isothermal atmosphere not only to allow purely oscillatory discrete modes to exist, but also to hold the inertia-acoustic and inertia-gravity waves a sufficient length of time for the weakly nonlinear energy exchanges analyzed in section 5 to occur, as will be further discussed in section 6 .
} 
the linearized version of (16)-(20) obtained by KQ00 are given by

$$
\left[\begin{array}{c}
u^{\prime}(\lambda, \phi, z, t) \\
v^{\prime}(\lambda, \phi, z, t) \\
w^{\prime}(\lambda, \phi, z, t) \\
p^{\prime}(\lambda, \phi, z, t) \\
\theta^{\prime}(\lambda, \phi, z, t)
\end{array}\right]=\left[\begin{array}{c}
\rho_{0}^{-1 / 2} U(\phi) \xi(z) \\
\rho_{0}^{-1 / 2} i V(\phi) \xi(z) \\
\rho_{0}^{-1 / 2} i P(\phi) \eta(z) \\
\rho_{0}^{1 / 2} P(\phi) \xi(z) \\
\rho_{0}^{1 / 2} P(\phi) \Theta(z)
\end{array}\right] e^{i s \lambda-i \sigma t}
$$

with the vertical structure functions $\xi(z), \eta(z)$, and $\Theta(z)$ satisfying the eigenvalue problem

$$
\begin{aligned}
\sigma \xi\left(\frac{1}{g H_{e}}-\frac{1}{C_{s}^{2}}\right)+\left(\frac{d \eta}{d z}-\Gamma \eta\right) & =0, \\
-\sigma \Theta+N^{2} \eta & =0, \\
\sigma \eta+\left(\frac{d \xi}{d z}+\Gamma \xi\right)-\Theta & =0,
\end{aligned}
$$

and the meridional structure functions $U(\phi), V(\phi)$, and $P(\phi)$ corresponding to the so-called Hough vector harmonics (Hough 1898; Longuet-Higgins 1968), which are the eigensolutions of the Laplace's tidal equations

$$
\begin{gathered}
-\sigma U-f V+\frac{s P}{a \cos \phi}=0, \\
\sigma V+f U+\frac{1}{a} \frac{d P}{d \phi}=0, \\
\frac{1}{a \cos \phi}\left[s U+\frac{d(V \cos \phi)}{d \phi}\right]=\frac{\sigma P}{g H_{e}}, \\
P=0 \quad \text { at } \quad \phi=-\pi / 2 \text { and } \quad \phi=\pi / 2 .
\end{gathered}
$$

In (26)-(28),

$$
\Gamma=\frac{g}{C_{S}^{2}}+\frac{1}{2 \rho_{0}} \frac{d \rho_{0}}{d z}=\frac{1-2 \kappa}{2 H}
$$

is the parameter of adiabatic expansion, where the second equality holds for the isothermal background atmosphere considered here, and $H_{e}$ is the separation constant, also known as equivalent height from the hydrostatic primitive-equation context (Taylor 1936). The solution of the vertical eigenvalue problem, (26)-(28), obtained by KQ00 is given by

$$
\begin{aligned}
& \eta(z)=A_{k} \sin (\hat{k} z), \\
& \xi(z)=\left(\frac{1}{C_{s}^{2}}-\frac{1}{g H_{e}}\right)^{-1} A_{k}[\hat{k} \cos (\hat{k} z)-\Gamma \sin (\hat{k} z)],
\end{aligned}
$$

$$
\Theta(z)=\frac{N^{2}}{\sigma} A_{k} \sin (\hat{k} z) .
$$

In (26)-(35) above, $A_{k}$ is an arbitrary constant and the eigenvalue $\hat{k}$ is written as

$$
\hat{k}^{2}=\left(\frac{1}{g H_{e}}-\frac{1}{C_{s}^{2}}\right)\left(N^{2}-\sigma^{2}\right)-\Gamma^{2} .
$$

The boundary conditions $\eta=0$ at $z=0$ and at $z=z_{T}$ imply that the vertical eigenvalues be quantized according to the relation

$$
\hat{k}=\frac{k \pi}{z_{T}} ; \quad k=1,2,3, \ldots,
$$

where $k$ is an integer characterizing the vertical mode. Thus, to compute a particular eigenmode, which is characterized by a zonal wavenumber $s$, a vertical mode index $k$, and a meridional quantum number $l$, and the oscillation type characterized by the corresponding eigenfrequency $\sigma$, one needs to evaluate the vertical eigenvalue from (37) and then solve (36) and the Laplace's tidal equations, (29)-(32), as a coupled eigenvalue problem to find the equivalent height $H_{e}$ and the eigenfrequency $\sigma$. Since the characteristic equation to determine the eigenvalue of the Laplace's tidal equations for a particular value of $H_{e}$ is transcendental, one needs to solve the coupled eigenvalue problem to find $H_{e}$ and $\sigma$ iteratively. In fact, isolating $\sigma$ in (36) leads to

$$
\sigma^{2}=N^{2}-\frac{g H_{e} C_{s}^{2}\left(\hat{k}^{2}+\Gamma^{2}\right)}{C_{s}^{2}-g H_{e}} .
$$

On the other hand, the characteristic equation to find $\sigma$ as an eigenvalue of the Laplace's tidal equation can be written schematically as follows:

$$
\sigma=F\left(H_{e}, s, l\right),
$$

with $F$ representing a transcendental monotonically growing function of $H_{e}$. KQ00 proposed two iterative methods to solve system (38) and (39) from suitable initial guesses, and we have implemented these iterative methods here. For details of the methods, see KQ00. In short, the basic idea is to iteratively find the value of $H_{e}$ for which the eigenfrequencies evaluated from (38) and (39) coincide with each other within a prespecified tolerance. As the eigenfrequency in (38) is a monotonically decreasing function of $H_{e}$, this solution always exists.

KQ00 labeled the possible solutions of system (38) and (39) as (i) oscillations of the first kind, corresponding to the pair of inertia-gravity modes (eastward and westward propagating), (ii) oscillations of the second 
kind, representing westward-propagating rotational modes or Rossby-Haurwitz waves, and (iii) oscillations of the third kind, corresponding to westward and eastward inertia-acoustic modes.

Unlike the hydrostatic primitive-equation model in which the equivalent height is constant for all the eigenmodes having the same vertical structure, in the present nonhydrostatic model the parameter $H_{e}$, apart from its dependence on the vertical mode $k$, also relies on the zonal wavenumber $s$, the meridional quantum number $l$, and the oscillation type. Furthermore, the parameter $H_{e}$ is useful to distinguish the inertia-acoustic and inertiagravity oscillation regimes in the present model. In fact, (36) shows that for $H_{e}>C_{s}^{2} / g$, it follows that $\sigma^{2}>N^{2}$, characterizing the acoustic regime. In contrast, $H_{e}<C_{s}^{2} / g$ implies that $\sigma^{2}<N^{2}$, which characterizes the gravity wave regime. The critical value $H_{\mathrm{ext}}=C_{s}^{2} / g$ refers to the equivalent height of the external mode. For this value of the equivalent height, the vertical velocity is null. Thus, in this case the inertia-acoustic modes are absent, and the inertia-gravity and rotational modes are identical to their hydrostatic counterparts (KQ00).

Figure 1 displays the dispersion curves of inertiaacoustic and inertia-gravity modes referred to the vertical mode $k=1$ and the meridional mode $l=0$. The meridional quantum index $l=0$ refers to the first symmetric mode in which the fluctuations $u^{\prime}, w^{\prime}, p^{\prime}, \rho^{\prime}$, and $\theta^{\prime}$ are even functions about the equator and the meridional velocity fluctuation $v^{\prime}$ is an odd function about the equator. One notices in Fig. 1 that for the vertical mode $k=1$ there exists a clear separation between the time frequencies of acoustic and gravity modes, since the internal inertia-gravity modes with the $k=1$ vertical structure have time frequencies much smaller than the Brunt-Väisälä frequency, whereas the correspondent inertia-acoustic modes have time frequencies relatively higher than the Brunt-Väisälä frequency. This time-scale separation between inertia-acoustic and inertia-gravity waves enhances for higher vertical modes (figures not shown). In fact, the inertia-acoustic (inertia-gravity) modes satisfy the relation $C_{s}^{2}-g H_{e}<0\left(C_{s}^{2}-g H_{e}>0\right)$, hence (36) shows that the higher the vertical eigenvalue, the higher the difference between the inertiaacoustic (inertia-gravity) mode eigenfrequency and the Brunt-Väisälä frequency.

Figure 2 displays the corresponding equivalent heights of the acoustic and gravity modes displayed in Fig. 1. One notices that the wide-scale separation between the eigenfrequencies of acoustic and gravity modes also reflects on the equivalent heights. In fact, Fig. 2 shows that the inertia-acoustic modes exhibit very large values of $H_{e}$ in the longwave region of the spectrum, while the

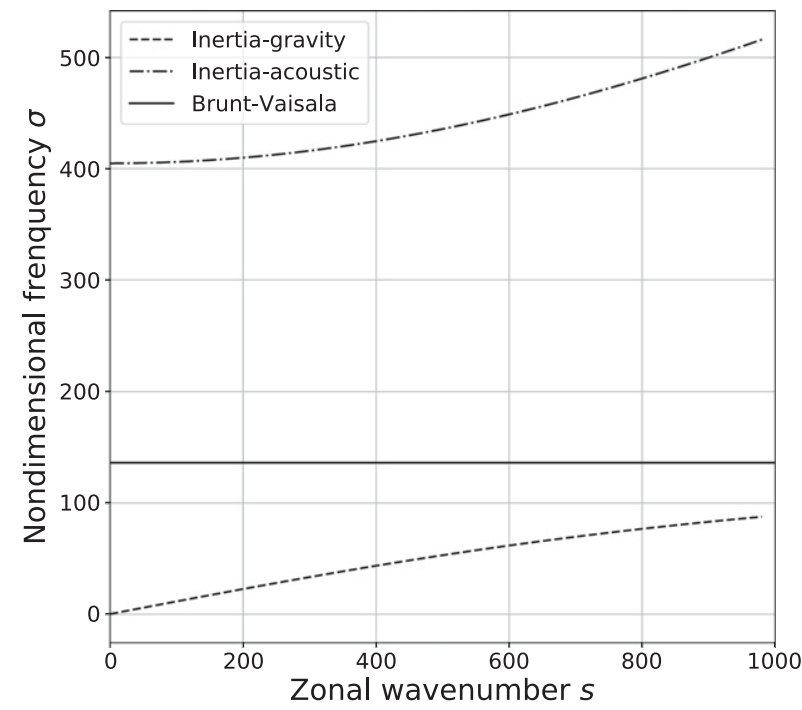

FIG. 1. Dispersion curves of inertia-acoustic (dotted-solid line) and inertia-gravity (dashed line) modes referred to the vertical mode index $k=1$ and the meridional quantum index $l=0$ (first symmetric meridional mode). The solid line refers to the BruntVäisälä frequency. The frequencies displayed here are nondimensionalized by $2 \Omega$.

equivalent height of acoustic modes asymptotically matches the equivalent height of the external mode for very large zonal wavenumbers. In contrast, the equivalent height of the internal inertia-gravity modes slightly decreases with the zonal wavenumber.

\section{Pseudoenergy conservation and orthogonality relation}

From the perturbation equations, (16)-(20), it follows that the pseudoenergy conservation ${ }^{3}$ of model equations, (1)-(6), can be written according to

$$
E^{(2)}+\text { h.o.t. }=\text { const, }
$$

where h.o.t. represents higher-order contributions, in terms model perturbation variables, to total pseudoenergy and $E^{(2)}$ refers to its leading-order contribution, given by

$$
\begin{aligned}
E^{(2)}= & \frac{1}{2} \int_{0}^{z_{T}} \int_{0}^{2 \pi} \int_{-\pi / 2}^{\pi / 2}\left[\rho_{0}\left(u^{\prime 2}+v^{\prime 2}+w^{\prime 2}\right)+\frac{p^{\prime 2}}{\rho_{0} C_{s}^{2}}\right. \\
& \left.+\frac{\theta^{\prime 2}}{\rho_{0} N^{2}}\right] a^{2} \cos \phi d \phi d \lambda d z .
\end{aligned}
$$

\footnotetext{
${ }^{3}$ Pseudoenergy refers to the disturbance energy, that is, the energy related to the departure from a reference steady state.
} 


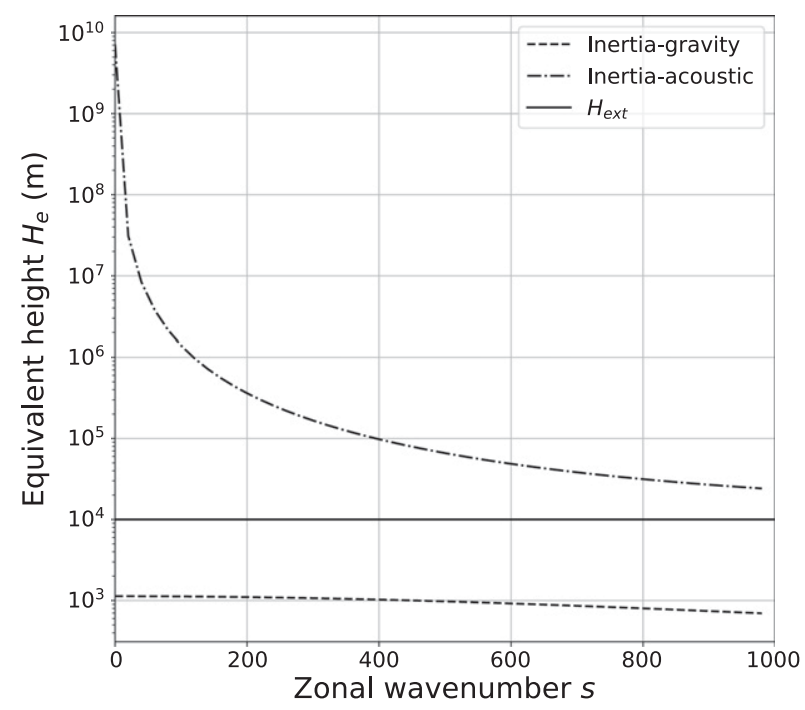

FIG. 2. Equivalent heights of inertia-acoustic (dotted-solid line) and inertia-gravity (dashed line) modes as a function of the zonal wavenumber. Both wave types displayed here have the $k=1$ vertical structure and the first symmetric meridional eigenstructure $(l=0)$. The solid line refers to the equivalent height of the external mode.

The disturbance energy densities inside the integral in (41) correspond, from left to right, to the kinetic, elastic, and thermobaric energies (Eckart 1960). A more accurate form of pseudoenergy conservation, which implies the explicit definition of h.o.t. in (40), is usually obtained by adopting Hamiltonian description of fluid dynamics equations in that pseudoenergy is obtained by combining the Hamiltonian conservation and the Casimir invariants (see, for instance, Scinocca and Shepherd 1992). Nevertheless, in both the linear and weakly nonlinear dynamics where the disturbances' amplitude is assumed to be small, $E^{(2)}$ is conserved (Domaracki and Loesch 1977; Raupp et al. 2008). Consequently, as we focus here on the weakly nonlinear normal mode interactions where only resonant triads are relevant, the explicit computation of only the leading-order component of the disturbance energy, $E^{(2)}$, is sufficient. Thus, the computation of h.o.t. in (40), which includes evaluating off-resonant triad interactions (Ripa 1981, 1983a,b; Vanneste and Vial 1994), is beyond the scope of this paper.

Besides the leading-order disturbance energy being conserved in either linear or weakly nonlinear contexts, for the resting background state considered here, characterized by a stably stratified atmosphere $\left(N^{2}>0\right)$, it follows that $E^{(2)}$ is also a positive definite integral. This is a sufficient condition for linear stability of normal mode solutions given by (25), according to Arnold's stability theorem (see Shepherd 1990, and references therein), with the eigenmodes describing neutral wave motions in this situation. This property has also been proved independently by KQ00 by demonstrating the following orthogonality relation for the normal modes:

$$
i\left(\sigma_{a}-\sigma_{b}\right)\left\langle\mathbf{u}_{a}, \mathbf{u}_{b}\right\rangle_{E^{(2)}}=0,
$$

where $\langle\ldots, \ldots\rangle_{E(2)}$ refers to the inner product in terms of pseudoenergy $E^{(2)}$, given by

$$
\begin{aligned}
\left\langle\mathbf{u}_{a}, \mathbf{u}_{b}\right\rangle_{E^{(2)}}= & \int_{0}^{z_{T}} \int_{0}^{2 \pi} \int_{-\pi / 2}^{\pi / 2}\left[\rho_{0}\left(u_{a}^{*} u_{b}+v_{a}^{*} v_{b}+w_{a}^{*} w_{b}\right)\right. \\
& \left.+\frac{p_{a}^{*} p_{b}}{\rho_{0} C_{s}^{2}}+\frac{\theta_{a}^{*} \theta_{b}}{\rho_{0} N^{2}}\right] a^{2} \cos \phi d \phi d \lambda d z
\end{aligned}
$$

In the equations above, $\mathbf{u}_{a}$ and $\mathbf{u}_{b}$ are vector functions representing two arbitrary linear eigenmodes of system (16)-(20) given by (25) and the superscript asterisk $(*)$ indicates the complex conjugate. Thus, (42) shows that the eigenvalues of the linearized version of (16)-(20) are purely imaginary (and, consequently, the eigenfrequencies are real, representing neutral oscillations) and the eigenvectors associated with distinct eigenvalues are orthogonal in terms of pseudoenergy. For $a=$ $b$, (42) defines the Hilbert norm of a linear eigenmode, given by

$$
E_{a}=\left\langle\mathbf{u}_{a}, \mathbf{u}_{a}\right\rangle_{E^{(2)}}>0 .
$$

The Hilbert norm $E_{a}$ defined above represents the intrinsic energy of an eigenmode $a$. In this case, the subscript $a$, which represents a particular eigensolution of the linearized version of (16)-(20), is composed of a zonal wavenumber $s$, a meridional quantum number $l$, a vertical mode index $k$, and a parameter $r$ that distinguishes the five possible oscillation types (westward and eastward inertia-gravity, westward and eastward inertia-acoustic and westward rotational modes).

As a consequence of the orthogonality relation (42), if one expands the solution of the perturbation equations, (16)-(20), in a normal mode series,

$$
\mathbf{u}=\left[u^{\prime}(\lambda, \phi, z, t), v^{\prime}(\lambda, \phi, z, t), w^{\prime}(\lambda, \phi, z, t), p^{\prime}(\lambda, \phi, z, t), \theta^{\prime}(\lambda, \phi, z, t)\right]^{\mathrm{T}}=\sum_{a} A_{a}(t) \mathbf{u}_{a},
$$


it follows that the leading-order disturbance energy has a diagonalized representation in terms of the normal modes

$$
E^{(2)}=\sum_{a}\left|A_{a}(t)\right|^{2} E_{a}
$$

The equation above represents the Parseval's identity for our model. According to (45), in the linear case, not only is the leading-order disturbance energy conserved, but its partition among the different eigenmodes is also unchanged in time [i.e., $|A(t)|^{2}$ is constant]. In contrast, in the weakly nonlinear regime, although $E^{(2)}$ is conserved, its partition among different eigenmodes may substantially vary in time due to the variation of $|A(t)|^{2}$ associated with the modes' coupling through resonant triads [see, for instance, Domaracki and Loesch (1977), Loesch and Deininger (1979), Vanneste and Vial (1994), and Raupp et al. (2008) for the hydrostatic context], as shall be demonstrated later. Consequently, before analyzing the energy exchanges among resonant modes in the weakly nonlinear regime, in order to clarify the physical implications of these energy exchanges, it is interesting to analyze first how the intrinsic mode energy $E_{a}$ varies with the zonal wavenumber for each mode type. In the next section, we will make this linear energy analysis for the inertia-gravity and inertia-acoustic mode types. Then, in section 5 we shall investigate the possibility of resonant interactions involving inertia-acoustic and inertia-gravity modes and analyze the possible physical implications of these triad interactions in view of the results presented in section 4. In both upcoming sections, we will focus on inertia-gravity and inertia-acoustic modes because the eigenfrequencies of the rotational modes are small, so that these modes are essentially unaffected by the nonhydrostatic effect of vertical acceleration.

\section{Linear energetics of inertia-gravity and inertia-acoustic modes}

The variation with zonal wavenumber of kinetic, elastic and thermobaric partitions of total intrinsic energy $E_{a}$ is depicted in Figs. 3 and 4 for inertia-acoustic and inertia-gravity modes, respectively. Figures 3 and 4 also display the spectral dependence of the three components of kinetic energy (zonal, meridional, and vertical kinetic energies). All the inertia-acoustic and inertia-gravity wave modes illustrated in Figs. 3 and 4 have the lowest meridional quantum index $l=0$ and the vertical mode index $k=1$. Thus, Figs. 3 and 4 refer to the acoustic and gravity branches of nonhydrostatic Kelvin wave, respectively (KQ00; Qian and Kasahara 2003). From Fig. 3 one notices that the total energy of inertiaacoustic modes is strongly dominated by elastic and

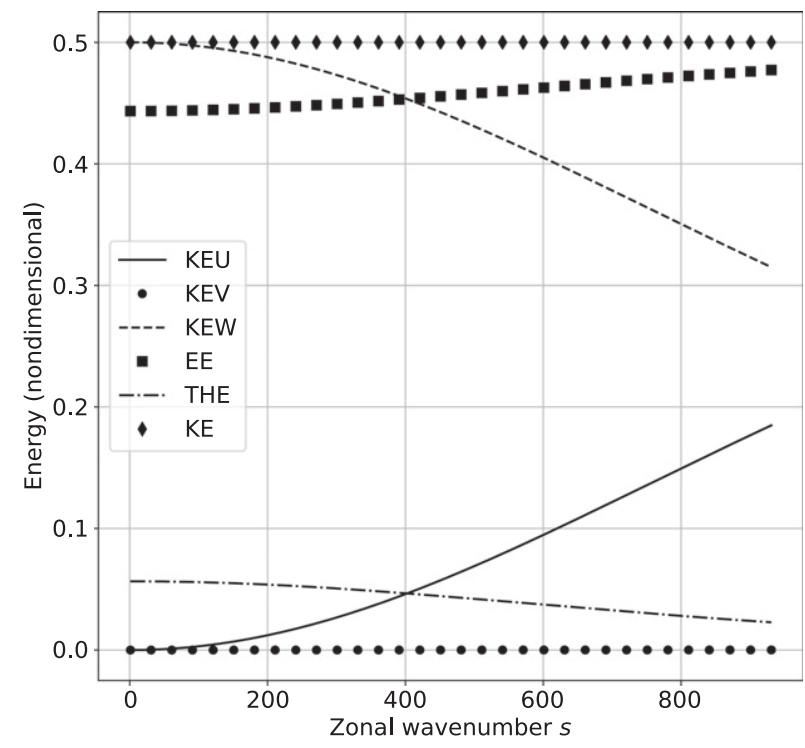

FIG. 3. Intrinsic energy norm of the $k=1$ and $l=0$ inertia-acoustic mode as a function of the zonal wavenumber, as well as its partition in kinetic (KE) [zonal (KEU), meridional (KEV), and vertical (KEW)], elastic (EE), and thermobaric energy (THE) types.

kinetic energies, as thermobaric energy is less than $10 \%$ of these dominant energy types. Furthermore, the kinetic energy is essentially associated with vertical motions, since the vertical kinetic energy is much higher than the horizontal ones, especially for planetary and large-scale modes. However, the zonal component of kinetic energy increases with zonal wavenumber so that for microscale waves $(s \approx 1000)$, the zonal kinetic energy becomes higher than thermobaric energy and of the same order as the vertical kinetic energy. Consequently, as we shall demonstrate in section 5, the energy exchanges between planetary-scale and small-scale inertia-acoustic modes due to the catalyst effect of small-scale gravity waves in the weakly nonlinear regime yield significant modulations in the zonal wind field.

Figure 4 shows that the energy of inertia-gravity modes is essentially associated with thermobaric and kinetic energies, since the elastic energy is less than $10 \%$ of the two dominant energy types. Moreover, for planetary and large-scale harmonics the kinetic energy is essentially due to its zonal component (recall that it is a Kelvin wave type, so the meridional velocity is negligible), since the vertical kinetic energy is negligible. However, the vertical kinetic energy of inertia-gravity modes increases with zonal wavenumber so that it becomes of the same order as the zonal kinetic energy for zonal wavenumber $s>700$. This reflects the nonhydrostatic effect of vertical acceleration on the inertiagravity modes. This effect is proportional to the time eigenfrequency of the mode. As the eigenfrequency of 


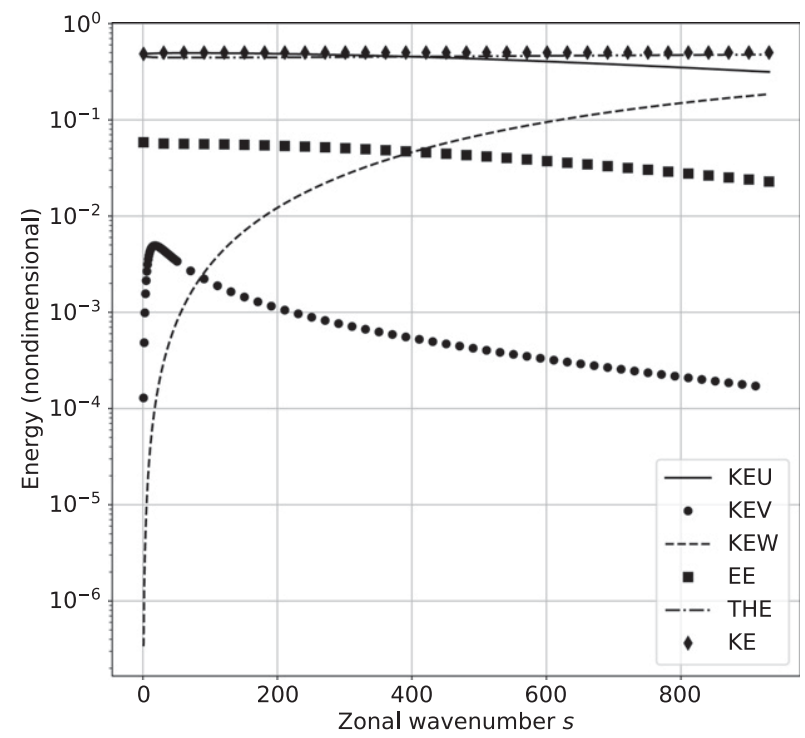

FIG. 4. As in Fig. 3, but for the inertia-gravity mode. Note the difference in the $y$-axis range.

inertia-gravity waves increases monotonically with zonal wavenumber (Fig. 1), the same occurs for the vertical acceleration and, thus, for the vertical kinetic energy. As a consequence of the spectral dependency of vertical kinetic energy of inertia-gravity wave modes, the energy modulations of small-scale gravity waves due to resonant interaction with small-scale and large- or planetary-scale acoustic modes can also yield significant vacillations in the vertical wind field, as shall be shown in the next section.

\section{Weakly nonlinear energetics of inertia-acoustic and inertia-gravity modes}

\section{a. Triad interaction equations}

Recall that in the weakly nonlinear regime the effect of nonlinearity is to break down the independence of normal modes, allowing their energy to be exchanged within resonant triads. Thus, to analyze the weakly nonlinear energetics of inertia-acoustic and inertiagravity wave modes in a simplified fashion, in this section we shall assume a highly truncated version of expansion (44) such that a single triad of normal modes is considered:

$$
\mathbf{u}=\left[\begin{array}{c}
u^{\prime}(\lambda, \phi, z, t) \\
v^{\prime}(\lambda, \phi, z, t) \\
w^{\prime}(\lambda, \phi, z, t) \\
p^{\prime}(\lambda, \phi, z, t) \\
\theta^{\prime}(\lambda, \phi, z, t)
\end{array}\right]=A_{1}(t) \mathbf{u}_{1}+A_{2}(t) \mathbf{u}_{2}+A_{3}(t) \mathbf{u}_{3}+\text { C.C. },
$$

where C.C indicates the complex conjugate of what is preceding, and the vector functions $\mathbf{u}_{j}, j=1,2,3$, represent a particular eigenmode of the linearized version of (16)-(20) given by (25) for $s=s_{j}, \sigma=\sigma_{j}, l=l_{j}$, and $k=k_{j}$. In this way, substituting the ansatz (46) into the perturbation equations, (16)-(20), and using the orthogonality relation in terms of pseudoenergy yield the so-called triad equations of the shallow global nonhydrostatic model:

$$
\begin{aligned}
& E_{1} \frac{d A_{1}}{d t}=\alpha_{1}^{23} A_{2} A_{3} e^{-i \delta t}, \\
& E_{2} \frac{d A_{2}}{d t}=\alpha_{2}^{13} A_{1} A_{3}^{*} e^{i \delta t}, \\
& E_{3} \frac{d A_{3}}{d t}=\alpha_{3}^{12} A_{1} A_{2}^{*} e^{i \delta t} .
\end{aligned}
$$

In the equations above, $\delta=\sigma_{1}-\sigma_{2}-\sigma_{3}$ is the time frequency mismatch among the triad components, $E_{j}, j=$ $1,2,3$, refers to their Hilbert norm given by (43), and the coupling constants $\alpha_{1}^{23}, \alpha_{2}^{13}$, and $\alpha_{3}^{12}$ represent the projection of the leading-order (quadratic) nonlinear terms of the perturbation equations due to the action of two wave modes onto another eigenmode; namely,

$$
\begin{aligned}
& \alpha_{1}^{23}=\left\langle\mathbf{u}_{1}, \mathbb{B}\left(\mathbf{u}_{2}, \mathbf{u}_{3}\right)+\mathbb{B}\left(\mathbf{u}_{3}, \mathbf{u}_{2}\right)\right\rangle_{E^{(2)}}, \\
& \alpha_{2}^{13}=\left\langle\mathbf{u}_{2}, \mathbb{B}\left(\mathbf{u}_{1}, \mathbf{u}_{3}^{*}\right)+\mathbb{B}\left(\mathbf{u}_{3}^{*}, \mathbf{u}_{1}\right)\right\rangle_{E^{(2)}}, \\
& \alpha_{3}^{12}=\left\langle\mathbf{u}_{3}, \mathbb{B}\left(\mathbf{u}_{1}, \mathbf{u}_{2}^{*}\right)+\mathbb{B}\left(\mathbf{u}_{2}^{*}, \mathbf{u}_{1}\right)\right\rangle_{E^{(2)}},
\end{aligned}
$$

where $\mathbb{B}$ is the bilinear operator containing the nonlinear terms of (16)-(20). The integrals involved in (50)-(52) are null unless the zonal wavenumbers and meridional indices satisfy certain selection rules. In the zonal direction, the orthogonality of the $e^{i s_{j} \lambda}$ components for different $j$ implies that the mode interaction takes place only if the zonal wavenumbers of the triad members satisfy the relation

$$
s_{1}=s_{2}+s_{3} .
$$

In contrast, as the meridional structure functions $U_{j}(\phi)$, $V_{j}(\phi)$, and $P_{j}(\phi)$ are obtained numerically, ${ }^{4}$ it is not possible to show an explicit selection rule for the meridional indexes unless asymptotic approximations are made. Vanneste and Vial (1994) examined the nonlinear wave interactions in the shallow-water primitive equations

\footnotetext{
${ }^{4}$ To compute the Hough functions for a particular value of $H_{e}$, we have used the numerical procedure described in Kasahara (1976) and Swarztrauber and Kasahara (1985).
} 
on the sphere and, in order to evaluate the interaction coefficients in a more analytic fashion, they considered the asymptotic form of the eigenfunctions in the limiting case of large equivalent height. In this limiting case, the Hough functions $U_{j}(\phi), V_{j}(\phi)$, and $P_{j}(\phi)$ are explicitly expressed in terms of spherical harmonics, and specific selection rules for all the possible kinds of triad interactions are shown. Analytic expressions of the nonlinear triad interaction coefficients and, consequently, explicit selection rules for the meridional coupling integrals in the shallow water primitive-equation context have also been obtained in the other limiting case of small equivalent heights (Ripa 1983a,b; Domaracki and Loesch 1977; Loesch and Deininger 1979). Explicit selection rules in terms of the meridional quantum indexes in spherical coordinate models have also been demonstrated in the context of Rossby wave interactions in the barotropic nondivergent model (Lynch 2009). Besides the meridional structure functions, the meridional integrals in (50)-(52) have also been computed numerically here by using the Gauss-Legendre quadrature formula. Nevertheless, the Hough vector harmonics can be either symmetric $\left(U_{j}\right.$ and $P_{j}$ even functions and $V_{j}$ an odd function about the equator) or antisymmetric $\left(U_{j}\right.$ and $P_{j}$ odd functions and $V_{j}$ an even function about the equator) (Kasahara 1977, 1976, 1978). Consequently, the only possibilities for the nonlinear interaction coefficients to be nonzero are two antisymmetric modes interacting with a symmetric one or three symmetric modes interacting.

In the vertical direction, the integrals in (50)-(52) appear in the form $\int_{0}^{z_{T}} \rho_{0}(z)^{-1 / 2} \cos \left[\left(k_{1} \pm k_{2} \pm k_{3}\right)\left(\pi z / z_{T}\right)\right] d z$. Such integrals are maximal if $k_{1} \pm k_{2} \pm k_{3}=0$, even though this condition is no longer excluding, in the sense that triads of normal modes whose vertical quantum numbers do not satisfy this relation might have smaller but nonzero nonlinear coupling constants. Consequently, the triads whose vertical mode indices satisfy the relation $k_{1} \pm$ $k_{2} \pm k_{3}=0$ are believed to undergo the most interactions, although this selection rule is not excluding as those associated with the zonal wavenumbers and the meridional structures. This nonexcluding selection rule of the vertical coupling integrals is also characteristic of the nonlinear wave interactions in the compressible primitive-equation model in isobaric coordinates (Raupp et al. 2008).

Apart from the selection rules discussed above, the energy exchanges among the triad components are maximized if the triad is resonant $(\delta=0)$. In this case, the mode energy equations obtained from (47)-(49) are given by

$$
E_{1} \frac{d\left|A_{1}\right|^{2}}{d t}=2 i \alpha_{1}^{23} \operatorname{Im}\left(A_{1}^{*} A_{2} A_{3}\right)
$$

$$
\begin{aligned}
& E_{2} \frac{d\left|A_{2}\right|^{2}}{d t}=2 i \alpha_{2}^{13} \operatorname{Im}\left(A_{1} A_{2}^{*} A_{3}^{*}\right), \\
& E_{3} \frac{d\left|A_{3}\right|^{2}}{d t}=2 i \alpha_{3}^{12} \operatorname{Im}\left(A_{1} A_{2}^{*} A_{3}^{*}\right) .
\end{aligned}
$$

From the equations above it follows that the total pseudoenergy conservation of the truncated equations, (47)-(49), implies that the nonlinear coupling constants of a resonant triad must satisfy the relation

$$
\alpha_{1}^{23}=\alpha_{2}^{13}+\alpha_{3}^{12} .
$$

\section{b. Numerical example for a resonance involving inertia-acoustic and inertia-gravity wave modes}

As the coupling constants $\alpha_{1}^{23}, \alpha_{2}^{13}$, and $\alpha_{3}^{12}$ are purely imaginary numbers, the dynamical system (47)-(49) is integrable by quadrature, with its analytical solution being expressed in terms of Jacobian elliptic functions (Craik 1986; Bustamante and Kartashova 2011). Assuming that mode 1 is the mode with the highest absolute value coupling coefficient and mode 3 the mode with the smallest one $\left(\left|\alpha_{1}^{23}\right|>\left|\alpha_{2}^{13}\right|>\left|\alpha_{3}^{12}\right|\right)$, for the initial condition $\left|A_{1}(t=0)\right|=0,\left|A_{2}(t=0)\right|=A_{2_{0}}$, and $\left|A_{3}(t=0)\right|=A_{3_{0}}$, the analytical solution of (47)-(49) in the resonant case $(\delta=0)$ can be written in terms of the modes' energies as follows (see, for instance, Domaracki and Loesch 1977; Raupp et al. 2008):

$$
\begin{aligned}
& E_{1}\left|A_{1}\right|^{2}(t)=E_{2} A_{2_{0}}^{2}\left|\frac{\alpha_{1}^{23}}{\alpha_{2}^{13}}\right| \operatorname{sn}^{2}(\tilde{T}, m), \\
& E_{2}\left|A_{2}\right|^{2}(t)=E_{2} A_{2_{0}}^{2} \operatorname{cn}^{2}(\tilde{T}, m), \\
& E_{3}\left|A_{3}\right|^{2}(t)=E_{3} A_{3_{0}}^{2} \operatorname{dn}^{2}(\tilde{T}, m),
\end{aligned}
$$

where "sn," "cn," and "dn" are the Jacobian elliptic functions, whose argument $\tilde{T}$ refers to the rescaled time, given by

$$
\tilde{T}=A_{3_{0}} \sqrt{\frac{\left|\alpha_{1}^{23} \alpha_{2}^{13}\right|}{E_{1} E_{2}}} t .
$$

In addition, the parameter $m$ of the Jacobian elliptic functions is given by

$$
m=\frac{\alpha_{3}^{12}}{\alpha_{2}^{13}} \frac{E_{2}}{E_{3}} \frac{A_{2_{0}}^{2}}{A_{3_{0}}^{2}} .
$$

A representative example of a set of three normal modes satisfying the selection rules (i) $s_{1}=s_{2}+s_{3}$, (ii) $k_{1}= \pm k_{2} \pm k_{3}$, and (iii) the integrands in (50)-(52) be even functions about the equator, as well as the 
TABLE 2. Representative example of a resonant triad involving inertia-acoustic(IA) and inertia-gravity (AG) modes. Apart from the modes' quantum indices, the table shows the numerical values of their correspondent eigenfrequencies, intrinsic energy norms, and coupling coefficients.

\begin{tabular}{crcccccc}
\hline \hline Mode $i$ & \multicolumn{1}{c}{$s$} & $l$ & $k$ & Kind & $\sigma_{i}(\mathrm{~Hz})$ & $E_{i}\left(\mathrm{~J} \mathrm{~kg}^{-1}\right)$ & $\alpha_{i}^{j k}\left(\mathrm{~J} \mathrm{~kg}^{-1}\right)$ \\
\hline 1 & 476 & 0 & 1 & IA & 0.062 & $5.17 \times 10^{10}$ & $-2.26 \times 10^{12} i$ \\
2 & 1 & 0 & 1 & IA & 0.058 & $5.27 \times 10^{20}$ & $-2.11 \times 10^{12} i$ \\
3 & 475 & 0 & 2 & IG & 0.004 & $2.8 \times 10^{7}$ & $-1.45 \times 10^{11} i$ \\
\hline
\end{tabular}

resonance condition $\sigma_{1} \approx \sigma_{2}+\sigma_{3}$, is displayed in Table 2. This triad interaction involves two inertiaacoustic modes (modes 1 and 2) and one inertia-gravity mode (mode 3 ). Mode 1 is a short-wavelength inertiaacoustic wave mode having zonal wavenumber $s_{1}=476$ and vertical mode index $k_{1}=1$; mode 2 is a planetaryscale inertia-acoustic wave mode with zonal wavenumber $s_{2}=1$ and vertical mode index $k_{2}=1$; mode 3 is a short-wavelength inertia-gravity wave mode with zonal wavenumber $s_{3}=475$ and vertical quantum number $k_{3}=2$. All the three modes are characterized by a meridional quantum index $l=0$, which corresponds to the first symmetric meridional eigenmode. According to (58)-(62), except for the special case of $m=1$, the solution of the triad equations is periodic in time, with the period of energy oscillation being inversely proportional to $A_{3_{0}}$. Also, the parameter $m$ relies on the ratio $A_{2_{0}} / A_{3_{0}}$ and, consequently, the period of energy oscillation can be arbitrarily long or short, depending on the initial mode amplitudes. Figures 5-7 illustrate the solutions (58)-(60) for the initial amplitudes $A_{2_{0}}$ and $A_{3_{0}}$ being set to yield physically reasonable values of the dynamical field perturbations $u^{\prime}, v^{\prime}, w^{\prime}, p^{\prime}$ and $\rho^{\prime}$.

Figure 5 shows the solution for $0<m \ll 1$. In this case, the amplitude of the inertia-gravity wave mode is much higher than the amplitudes of the two inertia-acoustic wave modes. Also, the energy of the inertia-gravity mode is almost unchanged in time. Consequently, the role of the inertia-gravity mode in this case is to essentially act as a catalyst mode for the energy exchanges between the two inertia-acoustic wave modes, which occur in a daily time scale. For higher values of the planetary acoustic mode initial energy and much smaller values of the gravity mode initial energy, the solution falls in the regime $0<m<1$. In this regime, as illustrated in Fig. 6, the three modes undergo energy oscillations, whose period is on the order of a few days. When the parameter $m$ is close to 1 (Fig. 7), mode 1 is rapidly excited at the expense of the energy loss of modes 2 and 3 , and its energy remains constant and dominates the total triad energy during the most part of the total interaction period.

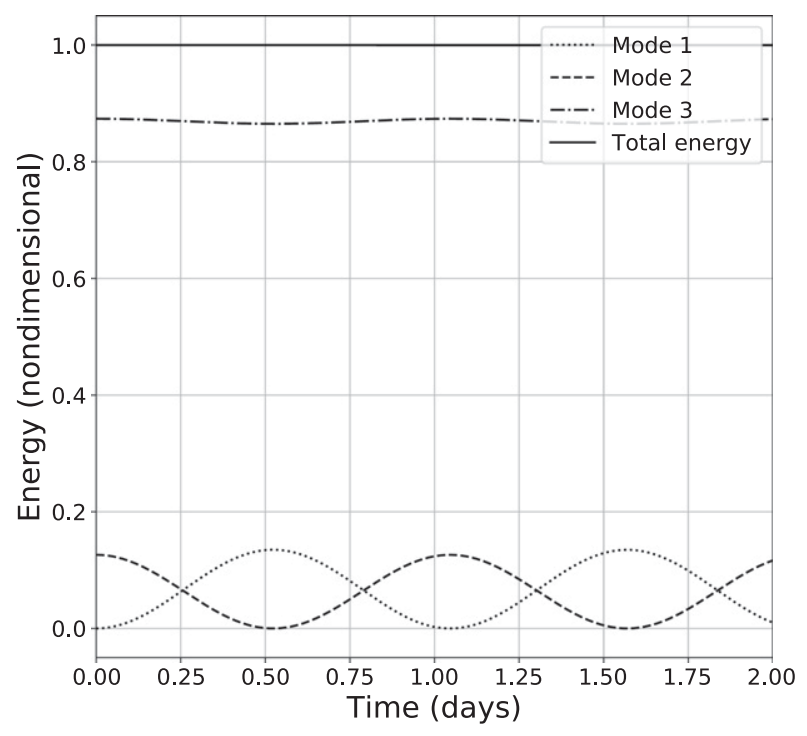

FIG. 5. Time evolution of the mode energies associated with the solutions (58)-(60) for the triad composed of an inertia-acoustic mode with zonal wavenumber $s=476$ and vertical mode index $k=$ 1 (mode 1), an inertia-acoustic mode with zonal wavenumber $s=1$ and vertical mode index $k=1$ (mode 2$)$, and an inertia-gravity mode with zonal wavenumber $s=475$ and vertical mode index $k=$ 2 (mode 3 ). All the modes have the first symmetric mode meridional structure $(l=0)$. The initial amplitudes of modes 2 and 3 are set to yield the parameter $m$ of the Jacobian elliptic functions close to 0 (see text for explanation).

The consequences of the modal energy exchanges for the dynamical field variables $u^{\prime}, w^{\prime}, p^{\prime}$, and $\rho^{\prime}$ are more pronounced in the regimes displayed in Figs. 6 and 7 than in the regime illustrated in Fig. 5. In fact, in the former case are alternations in the energy dominance among different eigenmodes, while in the latter regime the energy of the inertia-gravity mode remains always much higher than the energy of the two inertia-acoustic modes. Physical space manifestations of the nonlinear triad interaction illustrated in Fig. 7 are displayed in Figs. 8-10, which show the time evolution of the zonal and vertical wind perturbations at certain points of the domain. Figure 8 shows the time evolution of the zonal wind perturbation $u^{\prime}$ at an arbitrary longitude along the equator and the vertical level $z \approx 13 \mathrm{~km}$. As the zonal wind vertical structure associated with mode 3 vanishes at this vertical level (Fig. 11), the zonal wind perturbation displayed in Fig. 8 is due to the activity of the two inertia-acoustic modes of the triad. Furthermore, as discussed in the previous section, for the inertiaacoustic modes the zonal component of kinetic energy increases with the zonal wavenumber (Fig. 3), so the time evolution of the zonal wind perturbation displayed in Fig. 8 is strongly dominated by the short-wavelength acoustic mode of the triad (mode 1). In fact, comparing 


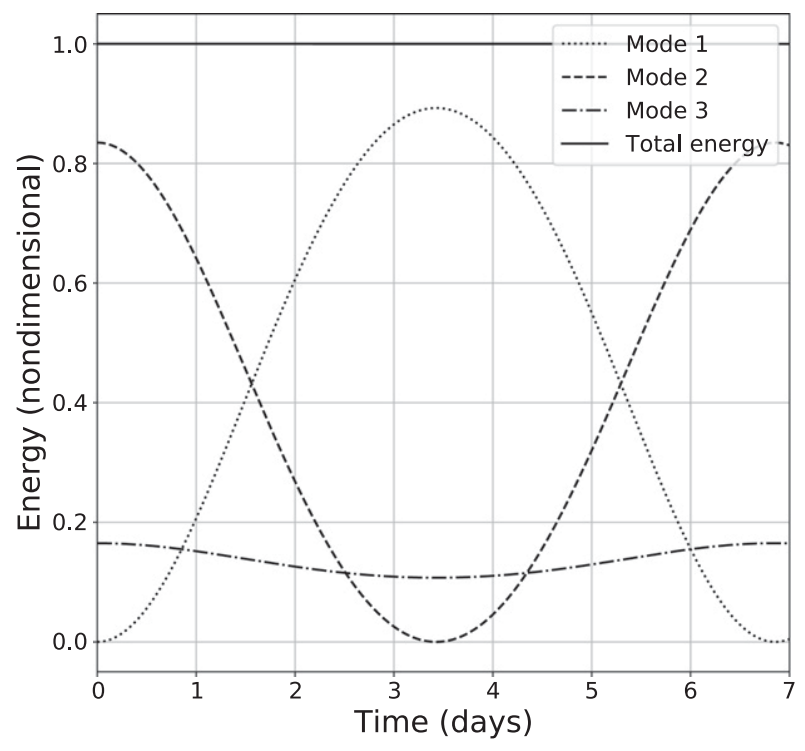

FIG. 6. As in Fig. 5, but for the initial amplitudes of modes 2 and 3 being set to yield $0<m<1$ (see text). Note the different time range on the $x$ axis.

Figs. 7 and 8 shows that the periods when mode 1 energy reaches a maximum (minimum) correspond to periods of higher (lower) amplitude oscillations of the zonal wind perturbations. The ultra-high-frequency oscillations embedded in the slowly varying amplitude modulations observed in Fig. 8 refer to the linear phase propagation of the two acoustic modes.

Similarly, the time evolution of the zonal wind perturbation at an arbitrary longitude along the equator and the vertical level $z \approx 8 \mathrm{~km}$ displayed in Fig. 9 is due to the activity of the inertia-gravity mode of the triad (mode 3 ), since the zonal wind vertical structure associated with modes 1 and 2 is null at this vertical level (Fig. 11). Consequently, the high-frequency oscillations of the zonal wind perturbation illustrated in Fig. 9 undergo amplitude modulations according to the gravity mode energy oscillation displayed in Fig. 7. Because the three modes have significant amount of their energy in the vertical kinetic form (see Figs. 3 and 4), the energy exchanges associated with the triad interaction analyzed here also yield significant modulations in the vertical wind perturbation, as illustrated in Fig. 10. Figure 10 shows the time evolution of $w^{\prime}$ at an arbitrary longitude along the equator and the vertical level $z \approx 14 \mathrm{~km}$. As the vertical structure function $\rho_{0}^{1 / 2} \eta(z)$ is maximal at this level for mode 3 but nonzero for modes 1 and 2 (Fig. 11), the three modes of the triad do contribute to the vertical wind fluctuations observed in Fig. 10. Since mode 1 strongly dominates the total triad energy during most part of the interaction period, the high-frequency oscillations of the vertical wind perturbation illustrated in

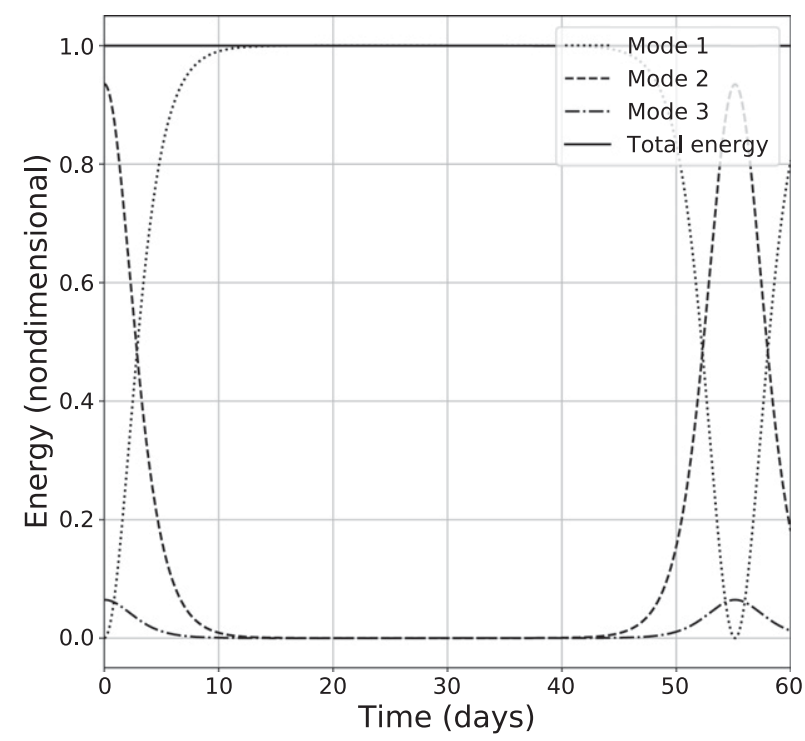

FIG. 7. As in Figs. 5 and 6, but for the initial amplitudes of modes 2 and 3 being set to yield $0 \ll m<1$ (see text). Note the different time range on the $x$ axis.

Fig. 10 undergo amplitude modulations according to mode 1 energy oscillation. However, the activity of modes 2 and 3 is evident during the periods of rapid growth and decay of the oscillation amplitude, being responsible for its two peaks before and after the amplitude plateau.

\section{Summary and conclusions}

Here we have extended the theory of global nonhydrostatic atmospheric normal modes by analyzing both their linear and weakly nonlinear energetics. The analysis has been performed by using the pseudoenergy orthogonality relation derived previously by KQ00. The linear analysis evidences the nonhydrostatic effect of vertical acceleration on the inertia-gravity wave modes. According to the results, for the inertia-gravity modes this nonhydrostatic effect increases with the zonal wavenumber and becomes evident for very short harmonics with zonal wavenumber $s>400$, as can be noticed through the spectral dependence of the vertical kinetic energy illustrated in section 4 . This feature justifies the hydrostatic approximation adopted in numerical models with resolutions coarser than around $10 \mathrm{~km}$. In addition, the linear energetic analysis demonstrates that the energy of the inertia-acoustic modes is essentially due to its elastic and kinetic components, with the latter being mostly caused by vertical motions. However, the zonal component of kinetic energy of the inertia-acoustic modes increases with the zonal wavenumber, so that for very short harmonics having zonal 


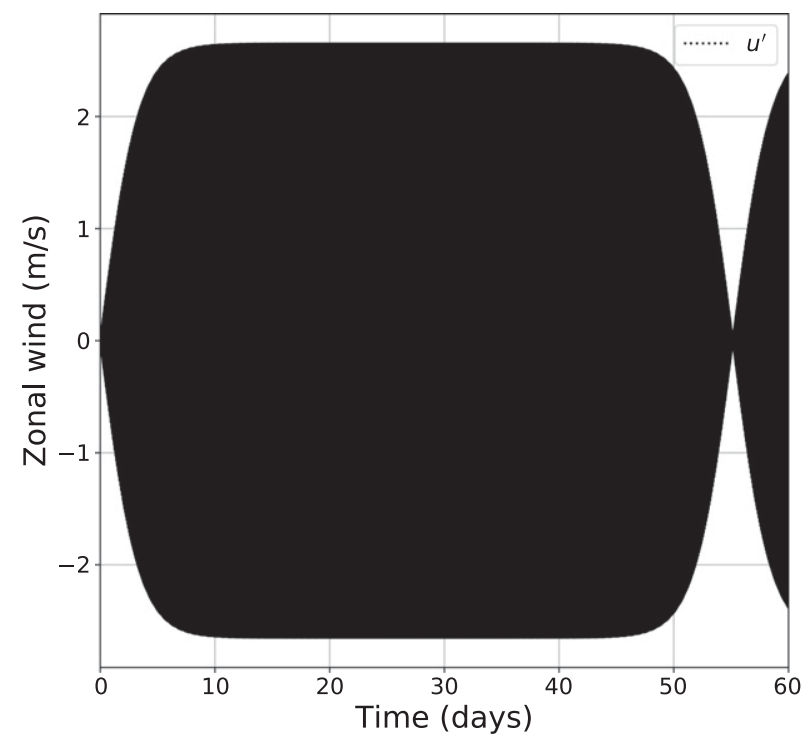

FIG. 8. Time evolution of the zonal wind perturbation $u^{\prime}$ at an arbitrary longitude along the equator and the vertical level $z \approx$ $13 \mathrm{~km}$, associated with the solution displayed in Fig. 7.

wavenumbers $s>700$, the zonal kinetic energy becomes of the same order as the vertical one. As a consequence, in the nonlinear regime the energy exchanges between planetary-scale and very short-wavelength inertia-acoustic modes in resonant triads involving a short-wavelength inertia-gravity mode yield strong amplitude modulations in the oscillations of the zonal wind perturbation.

With the resting background state considered here, characterized by a stably stratified atmosphere, the leading-order pseudoenergy is a positive definite integral, and consequently the linear eigenmodes represent purely stable wave motions according to Arnold's stability theorem. In this context, it is well known that the effect of nonlinearity is to break down the independence of the linear eigenmodes, allowing their leading-order pseudoenergy (which has a diagonalized representation in terms of the normal modes according to Parseval's identity) to be exchanged within resonant triads. Therefore, to investigate the weakly nonlinear energetics in a simplified fashion we have considered a highly truncated spectral expansion of the perturbation variables in terms of a single resonant triad composed of a short-wavelength inertia-acoustic mode, a planetaryscale inertia-acoustic wave mode and a short-wavelength inertia-gravity wave mode. The results have shown that an inertia-gravity mode essentially acts as a catalyst mode for the energy exchanges between two inertiaacoustic modes in a resonant triad, in the sense that it enables the interaction to occur and its amplitude level controls both the interaction period and the impacts of

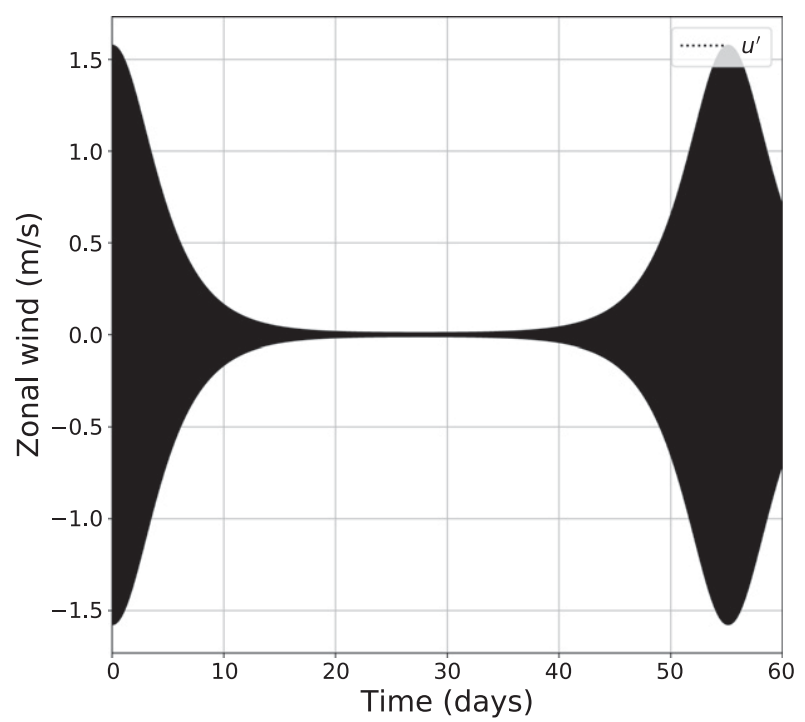

FIG. 9. Time evolution of the zonal wind perturbation $u^{\prime}$ at an arbitrary longitude along the equator and the vertical level $z \approx$ $8 \mathrm{~km}$, associated with the solution displayed in Fig. 7.

the energy exchanges for the dynamical field variables in the physical-space solution.

Thus, we have shown that the role of an inertiagravity mode in a resonant interaction with two inertiaacoustic modes in the nonhydrostatic dynamics is similar to the role of a Rossby mode in a resonant interaction with two inertia-gravity modes in the hydrostatic context. As the knowledge of the dynamics of nonlinear triad interaction involving two inertia-gravity modes and one rotational mode has provided important insights for the state of the art of the so-called nonlinear geostrophic adjustment problem (see, for instance, Duffy 1974; Vanneste and Vial 1994; Majda and Embid 1998; Vanneste and Yavneh 2004; Vanneste 2004; Hernandez-Duenas et al. 2014), the present work opens a new possible research line to investigate the role of these nonlinear interactions involving acoustic and nonhydrostatic gravity modes in the nonlinear hydrostatic adjustment. The hydrostatic adjustment problem has been studied in the linear context by Bannon (1995) and Duffy (2003) by adopting the assumption of horizontal homogeneity in which the equation of motion is considered only in the vertical direction. Both of these studies have pointed out the importance of vertically propagating acoustic modes for the adjustment process. Nevertheless, as the horizontal motions become of the same magnitude as the vertical ones for inertiaacoustic modes with zonal wavenumbers on the order of $s \approx 1000$ according to the linear energetic analysis presented here, further studies on the hydrostatic adjustment should be done by considering the horizontal dispersion as well, along with the effect of nonlinearity. 


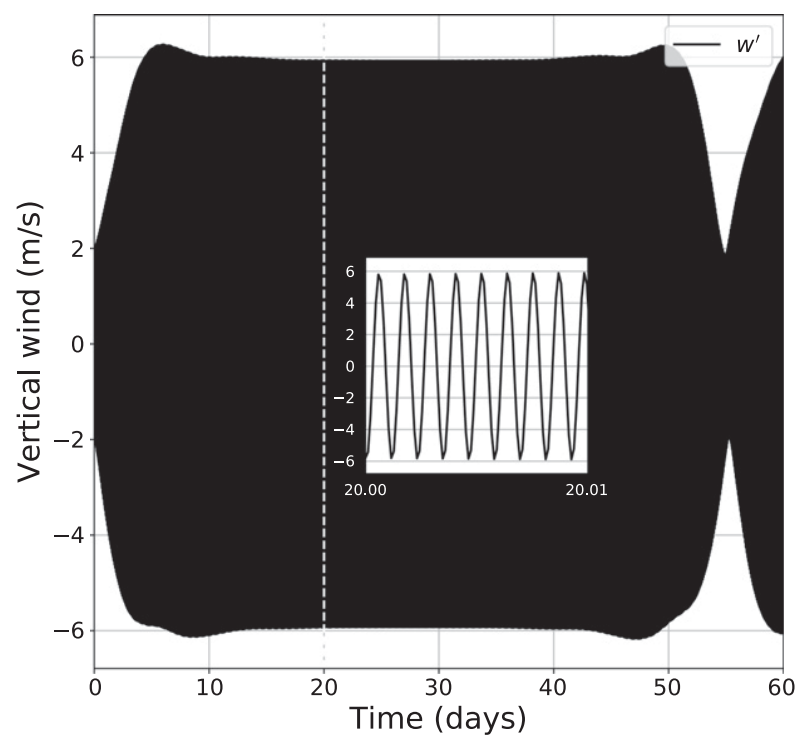

FIG. 10. Time evolution of $w^{\prime}$ at an arbitrary longitude along the equator and the vertical level $z \approx 14 \mathrm{~km}$, associated with the solution displayed in Fig. 7.

Another important point to be discussed is regarding the sensitivity of the results of our weakly nonlinear energetics analysis to the upper rigid lid assumption for the vertical boundary condition. The rigid lid approximation for the vertical boundary conditions has been commonly adopted in theoretical studies on tropospheric large-scale wave disturbances as the simplest model to explain observed wave speeds and structures (e.g., Khouider et al. 2013, and references therein). The justification for this assumption is based on the stronger stratification of the stratosphere in comparison with the troposphere, yielding inhibition of vertical motion at the tropopause. Nevertheless, as the ratio between the average static stability parameters of these two atmospheric layers is finite, a considerable amount of wave energy can radiate outward of the tropospheric domain. Chumakova et al. (2013) analyzed the effect of relaxing the upper rigid lid condition for internal gravity waves in the context of an incompressible, hydrostatic $(x, z)$-dependent flow in a nonrotating atmosphere. They showed that allowing an energy fraction to be transmitted to the stratosphere through their tropopause prototype yields discrete tropospheric eigenmodes having a damping with time scales ranging from an hour to a week. Consequently, in the simplified context of an isothermal background state adopted here, which implies a constant Brunt-Väisälä frequency, the upper rigid lid condition has a crucial role in holding the inertia-acoustic and inertia-gravity waves a sufficient length of time for the weakly nonlinear energy exchanges involving these two wave types to occur. Without the upper lid assumption,
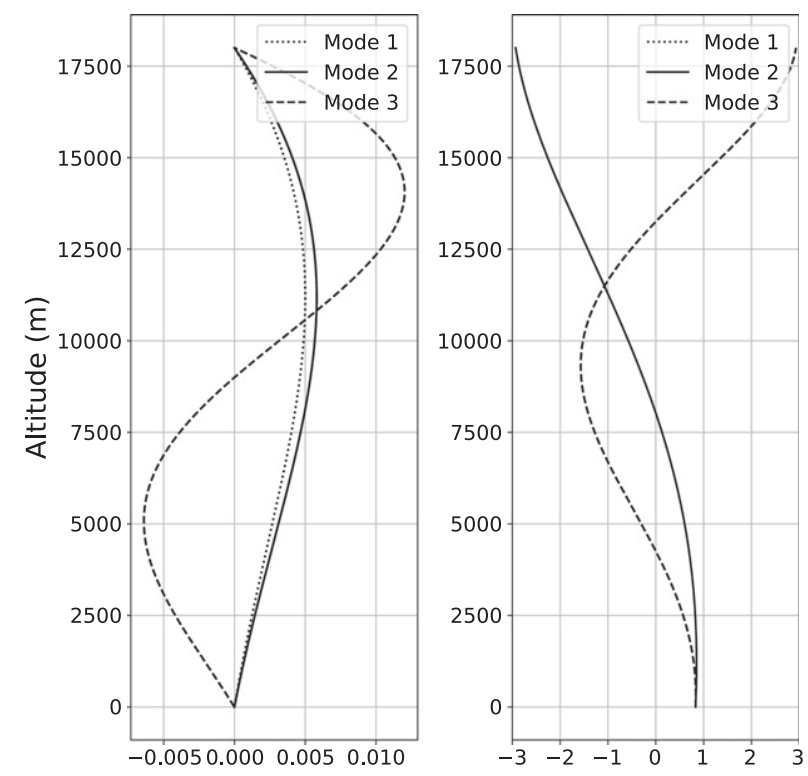

FIG. 11. Vertical structure functions of the zonal and vertical velocities associated with each mode of the triad. The subscripts of the vertical structure functions refer to the triad components.

the damping associated with the radiation of the waves would likely inhibit their nonlinear interaction.

Therefore, it could be inferred that the resonant interactions involving inertia-gravity and inertia-acoustic waves presented in section 5 might be at work in the atmosphere during conditions in which the stratospheric background state be characterized by a refraction index that traps both acoustic and gravity waves within the troposphere. For example, such refraction index can be associated with the background U.S. standard atmosphere temperature profile as demonstrated by linear WKB analysis for vertically propagating acoustic and nonhydrostatic gravity waves (Griffin and Thuburn 2018), so that the upper rigid lid condition can be thought of as a reasonable tool to mimic this trapping effect in our simplified model with an isothermal background atmosphere. However, as only seldom are the atmospheric waves embedded in a resting background state, the generalization of the present theory to more realistic scenarios should also include investigating the dynamics of the resonant nonlinear interactions between acoustic and nonhydrostatic gravity waves in the presence of critical layers associated with the propagation of these waves in vertically sheared flows.

Acknowledgments. Carlos F. M. Raupp and André Teruya were supported by Fundação de Amparo à Pesquisa do Estado de São Paulo (FAPESP) through Grant 2009/11643-4. André Teruya also received research scholarship from FAPESP through Grant 2012/06388-8. 
Carlos F. M. Raupp and Pedro L. Silva Dias are supported by FAPESP-PACMEDY through Grant 2015/50686-1.

\section{REFERENCES}

Baer, F., and J. Tribbia, 1977: On complete filtering of gravity modes through nonlinear initialization. Mon. Wea. Rev., 105, 1536-1539, https://doi.org/10.1175/1520-0493(1977)105<1536: OCFOGM $>2.0 . \mathrm{CO} ; 2$.

Bannon, P. R., 1995: Hydrostatic adjustment: Lamb's problem. J. Atmos. Sci., 52, 1743-1752, https://doi.org/10.1175/15200469(1995)052<1743:HALP > 2.0.CO;2.

Bustamante, M., and E. Kartashova, 2011: Resonance clustering in wave turbulent regimes: Integrable dynamics. Commun. Comput. Phys., 10, 1211-1240, https://doi.org/10.4208/cicp.110910.160211a.

Chumakova, L. G., R. R. Rosales, and E. G. Tabak, 2013: Leaky rigid lid: New dissipative modes in the troposphere. J. Atmos. Sci., 70, 3119-3127, https://doi.org/10.1175/JAS-D-12-065.1.

Craik, A. D. D., 1986: Wave Interactions and Fluid Flows. Cambridge University Press, 322 pp.

DeMaria, M., 1985: Linear response of a stratified tropical atmosphere to convective forcing. J. Atmos. Sci., 42, 1944-1959, https://doi.org/10.1175/1520-0469(1985)042<1944: LROAST $>2.0 . \mathrm{CO} ; 2$.

Domaracki, A., and A. Z. Loesch, 1977: Nonlinear interactions among equatorial waves. J. Atmos. Sci., 34, 486-498, https:// doi.org/10.1175/1520-0469(1977)034<0486:NIAEW>2.0.CO;2.

Duffy, D. G., 1974: Resonant interactions of inertio-gravity and Rossby waves. J. Atmos. Sci., 31, 1218-1231, https://doi.org/ 10.1175/1520-0469(1974)031<1218:RIOIGA > 2.0.CO;2.

—_ 2003: Hydrostatic adjustment in nonisothermal atmospheres. J. Atmos. Sci., 60, 339-353, https://doi.org/10.1175/ 1520-0469(2003)060<0339:HAINA $>2.0 . \mathrm{CO} ; 2$.

Eckart, C., 1960: Hydrodynamics of Oceans and Atmospheres. Pergamon Press, $290 \mathrm{pp}$.

Griffin, D. J., and J. Thuburn, 2018: Numerical effects on vertical propagation in deep-atmosphere models. Quart. J. Roy. Meteor. Soc., 144, 567-580, https://doi.org/10.1002/qj.3229.

Hernandez-Duenas, G., L. M. Smith, and S. N. Stechmann, 2014: Investigation of Boussinesq dynamics using intermediate models based on wave-vortical interactions. J. Fluid Mech., 747, 247-287, https://doi.org/10.1017/jfm.2014.138.

Holton, J., 2004: An Introduction to Dynamic Meteorology. Academic Press, $535 \mathrm{pp}$.

Hough, S. S., 1898: On the application of harmonic analysis to the dynamical theory of the tide-Part II: On the general integration of Laplace's dynamical equations. Philos. Trans. Roy. Soc. London, 191A, 139-185, https://doi.org/10.1098/rsta.1898.0005.

Kasahara, A., 1976: Normal modes of ultralong waves in the atmosphere. Mon. Wea. Rev., 104, 669-690, https://doi.org/ 10.1175/1520-0493(1976)104<0669:NMOUWI>2.0.CO;2.

_ 1977: Numerical integration of the global barotropic primitive equations with Hough harmonic expansions. J. Atmos. Sci., 34, 687-701, https://doi.org/10.1175/1520-0469(1977)034<0687: NIOTGB $>2.0 . \mathrm{CO} ; 2$.

_ 1978: Further studies on a spectral model of the global barotropic primitive equations with Hough harmonic expansions. J. Atmos. Sci., 35, 2043-2051, https://doi.org/10.1175/15200469(1978) $035<2043$ :FSOASM > 2.0.CO;2.

_ 2003a: On the nonhydrostatic atmospheric models with inclusion of the horizontal component of the Earth's angular velocity. J. Meteor. Soc. Japan, 81, 935-950, https://doi.org/ 10.2151/jmsj.81.935.

, 2003b: The roles of the horizontal component of the Earth's angular velocity in nonhydrostatic linear models. J. Atmos. Sci., 60, 1085-1095, https://doi.org/10.1175/1520-0469(2003) $60<1085$ :TROTHC $>2.0$. CO; 2 .

- , 2004: Free oscillations of deep nonhydrostatic global atmospheres: Theory and a test of numerical schemes. NCAR Tech. Rep. NCAR/TN-457+STR, 69 pp.

, and K. Puri, 1981: Spectral representation of threedimensional global data by expansion in normal mode functions. Mon. Wea. Rev., 109, 37-51, https://doi.org/10.1175/ 1520-0493(1981)109<0037:SROTDG >2.0.CO;2.

— , and J.-H. Qian, 2000: Normal modes of a global nonhydrostatic atmospheric model. Mon. Wea. Rev., 128, 3357-3375, https:// doi.org/10.1175/1520-0493(2000)128<3357:NMOAGN>2.0.CO;2.

Khouider, B., A. J. Majda, and S. Stechmann, 2013: Climate sciences in the tropics: Waves, vortices and PDEs. Nonlinearity, 26, R1, https://doi.org/10.1088/0951-7715/26/1/R1.

Leith, C. E., 1980: Nonlinear normal mode initialization and quasigeostrophic theory. J. Atmos. Sci., 37, 958-968, https://doi.org/ 10.1175/1520-0469(1980)037<0958:NNMIAQ>2.0.CO;2.

Lindzen, R. D., 1967: Planetary waves on beta-planes. Mon. Wea. Rev., 95, 441-451, https://doi.org/10.1175/1520-0493(1967) 095<0441:PWOBP $>2.3$.CO;2.

Loesch, A. Z., and R. C. Deininger, 1979: Dynamics of closed systems of resonantly interacting equatorial waves. J. Atmos. Sci., 36, 1490-1497, https://doi.org/10.1175/1520-0469(1979) 036<1490:DOCSOR >2.0.CO;2.

Longuet-Higgins, M. S., 1968: The eigenfunctions of Laplace's tidal equation over a sphere. Philos. Trans. Roy. Soc. London, 262, 511-607, https://doi.org/10.1098/rsta.1968.0003.

Lynch, P., 2009: On resonant Rossby-Haurwitz triads. Tellus, 61A, 438-445, https://doi.org/10.1111/j.1600-0870.2009.00395.x.

Machenhauer, B., 1977: On the dynamics of gravity oscillations in a shallow water model with applications to normal mode initialization. Beitr. Phys. Atmos., 50, 253-271.

Majda, A. J., and P. Embid, 1998: Averaging over fast waves for geophysical flows with unbalanced initial data. Theor. Comput. Fluid Dyn., 11, 155-169, https://doi.org/10.1007/s001620050086.

Matsuno, T., 1966: Quasi-geostrophic motions in the equatorial area. J. Meteor. Soc. Japan, 44, 25-43, https://doi.org/10.2151/ jmsj1965.44.1_25.

Pedlosky, J., 1987: Geophysical Fluid Dynamics. Springer-Verlag, 710 pp.

Phillips, N. A., 1973: Principles of large scale numerical weather prediction. Dynamic Meteorology, P. Morel, Ed., Springer, 1-96.

Qian, J.-H., and A. Kasahara, 2003: Nonhydrostatic atmospheric normal modes on beta-planes. Pure Appl. Geophys., 160, 1315-1358, https://doi.org/10.1007/s000240300007.

Raupp, C. F. M., and P. L. Silva Dias, 2009: Resonant wave interactions in the presence of a diurnally varying heat source. J. Atmos. Sci., 66, 3165-3183, https://doi.org/10.1175/2009JAS2899.1.

,-- E. G. Tabak, and P. Milewski, 2008: Resonant wave interactions in the equatorial waveguide. J. Atmos. Sci., 65 , 3398-3418, https://doi.org/10.1175/2008JAS2387.1.

Ripa, P., 1981: On the theory of nonlinear wave-wave interactions among geophysical waves. J. Fluid Mech., 103, 87-115, https:// doi.org/10.1017/S0022112081001250.

1983a: Weak interactions of equatorial waves in a one-layer model. Part I: General properties. J. Phys. Oceanogr., 13, 1208-1226, https://doi.org/10.1175/1520-0485(1983)013<1208: WIOEWI $>2.0 . \mathrm{CO} ; 2$. 
1983b: Weak interactions of equatorial waves in a onelayer model. Part II: Applications. J. Phys. Oceanogr., 13, 1227-1240, https://doi.org/10.1175/1520-0485(1983)013<1227: WIOEWI $>2.0 . \mathrm{CO} ; 2$.

Scinocca, J. F., and T. G. Shepherd, 1992: Nonlinear waveactivity conservation laws and Hamiltonian structure for the two-dimensional anelastic equations. J. Atmos. Sci., 49, 5-28, https://doi.org/10.1175/1520-0469(1992)049<0005: NWACLA $>2.0 . \mathrm{CO} ; 2$.

Shepherd, T. D., 1990: Symmetries, conservation laws, and Hamiltonian structure in geophysical fluid dynamics. Advances in Geophysics, Vol. 32, Academic Press, 287-338, https://doi.org/ 10.1016/S0065-2687(08)60429-X

Silva Dias, P. L., W. H. Schubert, and M. DeMaria, 1983: Large-scale response of the tropical atmosphere to transient convection. J. Atmos. Sci., 40, 2689-2707, https://doi.org/10.1175/1520-0469(1983)040<2689:LSROTT> 2.0.CO;2.

Swarztrauber, P. N., and A. Kasahara, 1985: The vector harmonic analysis of the Laplace's tidal equations. SIAM J. Sci. Stat. Comput., 6, 464-491, https://doi.org/10.1137/0906033.

Taylor, G. I., 1936: The oscillations of the atmosphere. Proc. Roy. Soc. London, 156, 318-326, https://doi.org/10.1098/ rspa.1936.0150.

Vallis, G. K., 2006: Atmospheric and Oceanic Fluid Dynamics. Cambridge University Press, 745 pp.
Vanneste, J., 2004: Inertia-gravity wave generation by balanced motion: Revisiting the Lorenz-Krishnamurthy model. J. Atmos. Sci., 61, 224-234, https://doi.org/10.1175/15200469(2004)061<0224:IWGBBM>2.0.CO;2.

- and F. Vial, 1994: Nonlinear wave propagation on a sphere: Interaction between Rossby waves and gravity waves; stability of the Rossby waves. Geophys. Astrophys. Fluid Dyn., 76, 121-144, https://doi.org/10.1080/03091929408203662.

_ , and I. Yavneh, 2004: Exponentially small inertia-gravity waves and the breakdown of quasigeostrophic balance. J. Atmos. Sci., 61, 211-223, https://doi.org/10.1175/15200469(2004)061<0211:ESIWAT>2.0.CO;2.

White, A. A., B. J. Hoskins, I. Roulstone, and A. Staniforth, 2005: Consistent approximate models of the global atmosphere: Shallow, deep, hydrostatic, quasi-hydrostatic and non-hydrostatic. Quart. J. Roy. Meteor. Soc., 131, 2081-2107, https:// doi.org/10.1256/qj.04.49.

Žagar, N., N. Gustafsson, and E. Källén, 2004: Variational data assimilation in the tropics: The impact of a background-error constraint. Quart. J. Roy. Meteor. Soc., 130, 103-125, https:// doi.org/10.1256/QJ.03.13.

, A. Kasahara, K. Terasaki, J. Tribbia, and H. Tanaka, 2015: Normal-mode function representation of global 3-D data sets: Open-access software for the atmospheric research community. Geosci. Model Dev., 8, 1169-1195, https://doi.org/10.5194/ gmd-8-1169-2015. 